\title{
Distribuição do tamanho de poros em um Latossolo Vermelho em sistema integrado de produção agropecuária
}

Rafael Luís Sanchez Perusso, Carolina dos Santos Batista Bonini, Cleiton Alexandre Silveira do Nascimento, Jéssica Fernanda Dias Souza, Gelci Carlos Lupatini, Hauan Andreozzi de Souza, Fabricio Gomes Pedro

Universidade Estadual Paulista - UNESP, Dracena, SP. E-mail: rafaelengenhar@gmail.com

\begin{abstract}
Resumo
Os sistemas integrados de produção agropecuária visam a sustentabilidade, procurando explorar o máximo da área, além de diminuir os impactos sobre as propriedades do solo. $O$ presente trabalho teve objetivo estudar a distribuição do tamanho de poros de um Latossolo Vermelho em um sistema integrado de produção agropecuária após três anos de implantação do sistema. $O$ experimento foi realizado na Fazenda pertencente ao grupo Facholi, no município de Caiuá/SP. O delineamento experimental utilizado foi em blocos casualizados, com três repetições e compostos por 7 tratamentos: 1 - Soja - Milho $+B$. ruziziensis (pastejo); 2 - Soja - Milho + Piatã (pastejo); 3 - Soja - Milho + Paiaguás (pastejo); 4 - Soja - Piatã (pastejo 2 anos) - Soja - Piatã (pastejo); 5 - Soja - Paiaguás (pastejo 2 anos) - Soja - Paiaguás (pastejo); 6 Soja - milho (Plantio direto); 7 - Soja - milho (Preparo convencional). Foram realizadas as avaliações dos seguintes atributos do solo: macroporosidade, microporosidade, porosidade total e densidade do solo, em duas camadas do solo; 0,00-0,10 e 0,10-0,20 m de profundidade, em três épocas de coleta, utilizando o teste Tukey a $5 \%$ de probabilidade para a comparação de médias. Os resultados mostram que a macroporosidade aumentou com o tempo ( 3 anos de avaliação), e houve uma diminuição da densidade do solo, mostrando que os sistemas avaliados influenciaram positivamente os atributos do solo estudados. De forma geral, todos ao tratamentos aumentaram a macroporosidade em relação a caracterização inicial, com destaque para os tratamentos soja+piatã e soja+milho+piatã, camada de $0-0,10 \mathrm{~m}$ que obtiveram valores médios superiores aos demais.
\end{abstract}

Palavras - chave: física do solo; matéria orgânica; densidade do solo.

\section{Distribution of size of pores in a Oxisol in an integrated system of agricultural production}

\begin{abstract}
Integrated agricultural production systems aim at sustainability, seeking to exploit the maximum of the area, as well as reducing impacts on soil properties. The objective of this work was to study the distribution of pore size of an Oxisol in an integrated agricultural production system after three years of system implantation. The experiment was carried out at Fazenda belonging to the Facholi group, in the municipality of Caiuá / SP. The experimental design was a randomized complete block with three replicates and composed of 7 treatments: 1 - Soya - Maize + B. ruziziensis (grazing); 2 - Soybean - Maize + Piatã (grazing); 3 - Soya - Maize + Paiaguás (grazing); 4 - Soya - Piatã (grazing 2 years) - Soya - Piatã (grazing); 5 Soybean - Paiaguás (grazing 2 years) - Soybean - Paiaguás (grazing); 6 - Soybean - maize (Direct planting); 7 - Soybean - corn (Conventional preparation). The following soil attributes were evaluated: macroporosity, microporosity, total porosity and soil density in two depths of soil; 0.00-0.10 and 0.10-0.20 m depth, in three sampling periods, using the Tukey test at $5 \%$ probability for the comparison of means. The results show that the macroporosity increased with time ( 3 years of evaluation), and there was a decrease in soil density, showing that the evaluated systems positively influenced the soil attributes studied. In general, all treatments increased the macroporosity in relation to the initial characterization, especially the soybean + piatan and soy + maize + piatã treatments, 0-0.10 m depth, which obtained higher, average values than the others.
\end{abstract}

Keywords: soil physics; organic matter; soil bulk density. 


\section{Introdução}

A compactação do solo causada pelo pisoteio animal tem sido apontada como uma das principais causas da degradação de áreas cultivadas em sistemas integrados de produção agropecuária (SIPAs) (CERVELATI et al., 2011; NASCIMENTO JUNIOR, 2017; SOUZA et al., 2019). $O$ intenso pisoteio animal no solo ocasiona pressão superior às máquinas agrícolas; entretanto, em camadas superficiais, geralmente nos primeiros 0,05 a 0,08 m (FLORES et al., 2007). A pressão exercida sobre o solo é o agente causador da compactação; entretanto, em sistemas conservacionistas sob SSD, a camada de palha na superfície do solo é grande atenuadora desse efeito.

O uso de SIPAs bem manejada, tem mostrado resultados interessantes em relação à compactação do solo (BONINI et al., 2016) que tem por característica reduzir a porosidade do solo, a macroporosidade que é modificado pelo manejo e com isso a densidade do solo e resistência mecânica a penetração também aumentam, o que pode prejudicar o desenvolvimento do sistema radicular das culturas, diminuindo consequentemente a produtividade (COSTA et al., 2015).

Porém, no Brasil, diversos produtores rurais ainda têm resistido a esta tecnologia devido às incertezas sobre o impacto dos animais nas propriedades físicas do solo e na produtividade das culturas agrícolas. No entanto, os resultados de pesquisa demonstraram que os SIPAs podem melhorar as propriedades físicas, químicas e biológicas do solo e aumentar os estoques de $C$ dependendo da intensidade de pastejo (CARVALHO et al., 2010; ASSMANN et al., 2014; COSTA et al., 2015). Aliado a isso, a adoção de SIPAs em maior escala diminuiria o desmatamento e a degradação das pastagens, principalmente no Cerrado e na Amazônia (MACEDO, 2009).

Sistemas conservacionistas de produção agrícola, como SIPAs, melhorando o ambiente para o desenvolvimento das culturas na área agrícola, como a inclusão de pastagens na rotação de culturas provoca melhoria da qualidade física do solo por causa da combinação de três efeitos principais: ausência de preparo durante o ciclo da pastagem, presença de um denso sistema radicular atuando como agente agregante e aumento da atividade da macrofauna do solo (MARCHÃO et al., 2007). Santos et al. (2007) e Sarmento et al. (2008) constataram que mais da metade da massa de raízes de espécies de braquiária e de colonião concentra-se na camada de 0-0,10 $\mathrm{m}$ do solo. Em trabalho realizado por Costa et al. (2015), avaliando diferentes tipos de consórcios e sua influência sobre as propriedades físicas do solo, verificaram que os sistemas consorciados de culturas graníferas com capins tropicais promoveram redução da compactação do solo refletindo no incremento da macroporosidade e porosidade total e diminuição da resistência mecânica a penetração e densidade do solo, nas camadas de $0-0,10$ e $0,10-0,20 \mathrm{~m}$.

Segundo Prado (2013), a porosidade é importante porque influi na dinâmica da água no solo representando o volume de espaços vazios entre as partículas mais finas até as mais grosseiras e em solos arenosos (por exemplo, Neossolos Quartzarênicos e Argissolos com textura arenosa no horizonte A) apresentam predominância de poros grandes (porosidade total próxima de $40-50 \%$ ). As propriedades físicas: a porosidade, o arejamento, a resistência à penetração e o teor de água são sempre interrelacionados influindo decisivamente no crescimento radicular. A densidade do solo por possuir estreita relação com outros atributos, a grande maioria das pesquisas converge para o fato de que, com o seu aumento, ocorre a diminuição da porosidade total, macroporosidade, condutividade hidráulica, absorção iônica, assim como o consequente aumento da microporosidade e da resistência mecânica à penetração do solo. Esse fato desencadeia, no geral, diminuição da produtividade agrícola (CHIODEROLI et al., 2012; COSTA et al., 2015; BONINI et al, 2016; COSTA et al., 2016; PARIZ et al., 2017)

Em trabalho realizado por Spera et al. (2009), objetivando estudar o manejo adequado do solo para fins conservacionistas, os autores afirmaram que o aumento de macroporos é o aspecto mais importante e o valor da macroporosidade menor que $0,10 \mathrm{~m} 3 \mathrm{~m}-3$ pode tornar-se crítico. Entretanto, conforme relatado por Lanzanova et al. (2007), em valores abaixo de $0,10 \mathrm{~m} 3 \mathrm{~m}-3$, não interferiram na produção das culturas utilizadas nos sistemas produtivos, tendo em vista que essas atingiram elevados valores de produção. Assim, para solos tropicais, esse limite de $0,10 \mathrm{~m} 3 \mathrm{~m}-3$ pode não ser o real valor limitante à produção agrícola (COSTA et al., 2015). 
Tendo em vista que após três anos de implantação, o sistema já tenha modificado o solo de forma positiva, melhorando assim toda a sua estrutura, objetivou-se então estudar a distribuição do tamanho de poros do solo em Latossolo Amarelo em sistema integrado de produção agropecuária.

\section{Material e Métodos}

O experimento foi realizado em uma área pertencente ao Grupo Facholi, localizada no município de Caiuá - SP, localizada na região sudoeste do Estado de São Paulo a altitude de 375 metros, latitude $21^{\circ} 49^{\prime} 58^{\prime \prime}$ Sul, longitude $51^{\circ} 59^{\prime} 24^{\prime \prime}$ Oeste. O clima da região é classificado como Clima tropical com estação seca, Classificação climática de Köppen-Geiger: Aw (ALVAREZ et al., 2013). A precipitação média anual é de $1.154 \mathrm{~mm}$ e a temperatura média anual é de $22.8^{\circ} \mathrm{C}$. O solo em estudo é um Latossolo Amarelo (SANTOS et al., 2018), com textura arenosa $\left(820 \mathrm{~g} \mathrm{~kg}^{-1}\right.$ de areia, $110 \mathrm{~g} \mathrm{~kg}^{-1}$ de argila e $70 \mathrm{~g} \mathrm{~kg}^{-1}$ de silte). Antes da implantação do experimento foi realizada a caracterização inicial das propriedades físicas da área experimental no ano de 2015 , com os resultados apresentados na tabela 1.

Tabela 1. Caracterização física da área experimental. Caiuá-SP, 2015.

\begin{tabular}{|c|c|c|c|}
\hline $\begin{array}{c}\text { Camada } \\
(\mathrm{m})\end{array}$ & $\begin{array}{c}\text { Ds } \\
\mathrm{g} \mathrm{cm}^{-3}\end{array}$ & $--\mathrm{cm}^{3} \cdot \mathrm{cm}^{-3}$ & $\begin{array}{l}\text { PT } \\
3 .--- \\
\end{array}$ \\
\hline $0-0,10$ & 1,55 & $0,05 \quad 0,19$ & 0,24 \\
\hline $0,10-0,20$ & 1,63 & $0,04 \quad 0,16$ & 0,20 \\
\hline
\end{tabular}

Taxa de infiltração $=62,90 \mathrm{~mm} \cdot \mathrm{h}^{-1}$

Foi utilizado o delineamento em blocos ao acaso, com os seguintes tratamentos: 1-Soja Milho + B. ruziziensis (pastejo); 2-Soja - Milho + Piatã (pastejo); 3-Soja-Milho + Paiaguás (pastejo); 4-Soja - Piatã (pastejo 2 anos) - Soja Piatã (pastejo); 5 - Soja - Paiaguás (pastejo 2 anos) - Soja - Paiaguás (pastejo); 6-Soja - milho (Plantio direto).

A área foi cultivada com soja com semeadura em novembro de 2015 e colheita em fevereiro de 2016. A adubação de implantação foi de $300 \mathrm{~kg} / \mathrm{ha}$ da fórmula 04-30-10. Em seguida, foi realizada a semeadura do milho sob sistema de plantio direto, juntamente com as forrageiras no dia 08/03/2016, utilizando o espaçamento de 0,9 $\mathrm{m}$ entre linhas para o milho e $0,4 \mathrm{~m}$ para o capim. Os capins utilizados foram Urochloa brizantha cv. Paiaguás, U. brizantha cv. Piatã e U. ruziziensis. $\mathrm{O}$ híbrido de milho utilizado foi o DKB 390 e a colheita foi realizada em agosto de 2016. A adubação de implantação foi $300 \mathrm{~kg} / \mathrm{ha}$ da fórmula 08-28-16. A aplicação de nitrogênio em cobertura não foi realizada devido ao período (abril) de deficiência hídrica. Na safra, 2016-17 foram feitas as culturas de soja (novembro/2016 a fevereiro/2017) e milho (fevereiro/2017 a agosto/2017).

Foram coletadas duas amostras (duplicatas) em cada parcela dos três blocos. Os anéis volumétricos de $100 \mathrm{~cm}^{3}$ foram realizadas com o auxílio de um trado para a coleta de amostras indeformadas. A porosidade total foi determinada pela saturação do solo (volume de poros totais do solo ocupado pela água), a microporosidade pelo método da mesa de tensão com coluna de água de $6 \mathrm{kPa}$ e a macroporosidade foi calculada por diferença entre a porosidade total e a microporosidade, e a densidade do solo pelo método do anel volumétrico de acordo com Teixeira et al. (2017), nas camadas de solo de $0-0,10 \mathrm{~m}$ e 0,10-0,20m, em 3 épocas distintas: setembro de 2016; fevereiro de 2017 e Novembro de 2017.

Os dados de tratamentos foi 0 tratamento principal e os anos de coletas as parcelas subdivididas, os mesmos foram submetidos à análise de variância e teste Tukey a $5 \%$ de probabilidade para a comparação de médias utilizando-se o programa estatístico Sisvar (FERREIRA, 2011).

\section{Resultados e Discussão}

Os valores médios, $\mathrm{CV}$ e teste $\mathrm{F}$ da porosidade estão apresentados na Tabela 2 . Observa-se que na camada de $0-0,10 \mathrm{~m}$ houve diferenças significativas entre os tratamentos para microporosidade, com o maior valor encontrado para o tratamento com rotação soja- 
milho em sistema de plantio direto, porem este maior valor de microporosidade não influenciou de forma significativa na porosidade total.

De acordo com Souza et al. (2019) em estudo feito sobre sistemas de produção de grãos, envolvendo pastagem sob sistema de plantio direto, verificaram diferenças de microporosidade entre os sistemas de manejo. Os valores encontrados para microporosidade no presente trabalho foram superiores a resultados encontrados por Marchão et al. (2007). Quando se compara os resultados ao longo do período de condução dos experimentos, pode-se observar que na primeira coleta realizada em setembro de 2016, após a colheita do milho, o valor para macroporosidade é menor e consequentemente, a microporosidade tem o maior valor.

$\mathrm{Na}$ segunda coleta realizada em fevereiro de 2017, após a colheita da soja, a macroporosidade passa a ter um valor maior, e a microporosidade um menor valor, ou seja, a distribuição de macro e microporos foi diretamente afetada de acordo com a cultura em questão (tabela 2). Esse efeito cíclico evidencia-se quando observa-se os valores obtidos na terceira coleta em novembro de 2017, novamente após a colheita da cultura do milho, quando os valores da distribuição dos poros voltam a se aproximar dos valores obtidos na primeira coleta.

Tabela 2. Médias para macroporosidade (\%), microporosidade (\%), porosidade total (\%) e densidade do solo $\left(\mathrm{kg} \mathrm{dm}^{-3}\right)$ para as camadas de 0,0-0,10m e 0,10-0,20m. Caiuá - SP. 2016-17.

\begin{tabular}{|c|c|c|c|c|}
\hline \multicolumn{5}{|c|}{$0-0,10 m$} \\
\hline Tratamento & Ma & Mi & $\mathbf{P t}$ & Ds \\
\hline Soja-Milho+U. ruziziensis & 17,21 & $20,85 \mathrm{~b}$ & 38,06 & 1,61 \\
\hline Soja-Milho+Piatã & 16,00 & $22,27 a b$ & 38,28 & 1,61 \\
\hline Soja-Milho+Paiaguás & 16,04 & $22,20 a b$ & 38,24 & 1,61 \\
\hline Soja-Piatã & 18,71 & $21,32 \mathrm{~b}$ & 40,03 & 1,56 \\
\hline Soja-Paiaguás & 17,11 & $22,31 a b$ & 39,43 & 1,56 \\
\hline Soja-Milho & 15,67 & $23,60 \mathrm{a}$ & 39,29 & 1,55 \\
\hline \multicolumn{5}{|l|}{ Ano } \\
\hline Set/2016 & $15,6 \mathrm{~b}$ & $26,69 \mathrm{a}$ & $42,29 a$ & $1,54 \mathrm{~b}$ \\
\hline Fev/2017 & $23,47 a$ & $17,73 \mathrm{c}$ & $41,21 \mathrm{a}$ & $1,61 \mathrm{a}$ \\
\hline Nov/2017 & $11,31 \mathrm{c}$ & $21,85 b$ & $33,16 \mathrm{~b}$ & $1,61 \mathrm{a}$ \\
\hline $\mathrm{F}$ (trat) & $0,52^{\mathrm{ns}}$ & $4,26^{*}$ & $0,38^{\text {ns }}$ & $1,29^{\text {ns }}$ \\
\hline $\mathrm{F}($ ano) & $46,02^{*}$ & $62,45^{*}$ & $60,21^{*}$ & $8,21^{*}$ \\
\hline $\mathrm{F}($ trat*ano) & $1,36^{\mathrm{ns}}$ & $0,52^{\text {ns }}$ & $1,47^{\text {ns }}$ & $0,97^{\text {ns }}$ \\
\hline CV (1) \% & 28,01 & 6,25 & 9,97 & 4,77 \\
\hline $\mathrm{CV}(2) \%$ & 22,96 & 10,89 & 7,01 & 3,85 \\
\hline \multicolumn{5}{|c|}{$0,10-0,20 m$} \\
\hline Tratamento & $\mathrm{Ma}$ & Mi & Pt & Ds \\
\hline Soja-Milho+U. ruziziensis & $15,04 \mathrm{~b}$ & 20,88 & $35,92 \mathrm{~b}$ & $1,66 \mathrm{a}$ \\
\hline Soja-Milho+Piatã & $13,83 \mathrm{~b}$ & 22,78 & $36,61 a b$ & $1,64 \mathrm{ab}$ \\
\hline Soja-Milho+Paiaguás & $14,44 \mathrm{~b}$ & 22,66 & $37,10 a b$ & $1,63 a b$ \\
\hline Soja-Piatã & 18,28 a & 21,51 & $39,80 \mathrm{a}$ & $1,58 \mathrm{~b}$ \\
\hline Soja-Paiaguás & $15,42 a b$ & 22,85 & $38,28 a b$ & $1,62 \mathrm{ab}$ \\
\hline Soja-Milho & $14,91 \mathrm{~b}$ & 22,19 & $37,10 a b$ & $1,63 \mathrm{ab}$ \\
\hline \multicolumn{5}{|l|}{ Ano } \\
\hline Set/2016 & $13,28 \mathrm{~b}$ & $27,15 \mathrm{a}$ & 40,43 a & $1,60 \mathrm{~b}$ \\
\hline Fev/2017 & 21,60 a & $18,06 \mathrm{c}$ & 39,66 a & $1,64 \mathrm{a}$ \\
\hline Nov/2017 & $11,08 \mathrm{c}$ & $21,23 \mathrm{~b}$ & $32,31 \mathrm{~b}$ & $1,64 \mathrm{a}$ \\
\hline $\mathrm{F}$ (trat) & $5,77^{*}$ & $1,01^{\mathrm{ns}}$ & $3,33^{*}$ & $2,97^{\text {ns }}$ \\
\hline $\mathrm{F}(\mathrm{ano})$ & $133,82^{*}$ & $36,05^{*}$ & $38,49^{*}$ & $5,87^{*}$ \\
\hline $\mathrm{F}$ (trat*ano) & $3,90^{*}$ & $1,20^{\mathrm{ns}}$ & $0,44^{\mathrm{ns}}$ & $0,97^{\mathrm{ns}}$ \\
\hline$C V(1) \%$ & 12,63 & 10,72 & 6,03 & 2,71 \\
\hline $\mathrm{CV}(2) \%$ & 13,29 & 14,72 & 8,18 & 2,74 \\
\hline
\end{tabular}

Médias seguidas por letras iguais não diferem estatisticamente pelo teste Tukey a $5 \%$ de probabilidade. 
Em estudo realizado por Chioderoli (2010) também foram encontrados resultados semelhantes aos obtidos na presente pesquisa na camada de $0-0,10 \mathrm{~m}$, onde a macroporosidade também aumentou após a colheita da soja quando comparada a coleta feita após a cultura do milho. Esse efeito da distribuição dos poros também acontece na camada mais profunda $(0,10-0,20)$ (Tabela 2). Resultados contrários a este trabalho foram verificados por Souza et al. (2019) que estudaram sistemas integrados após cinco anos de implantação.

Nas avaliações realizadas durante o decorrer dos experimentos, observa-se que a porosidade total diminuiu nas duas camadas estudadas, o que consequentemente influenciou também no aumento da densidade do solo (Tabela 2).

$\mathrm{Na}$ camada de 0,10-0,20 $\mathrm{m}$ houve diferença significativa para macroporosidade e porosidade total, sobressaindo-se o tratamento com a rotação de soja com a $U$. brizantha $c V$. Piatã para os dois atributos. Quando se compara os tratamentos Soja-Piatã e Soja-milho+U. Ruziziensis pode se observar que estes tem valores inversos de macroporosidade na camada mais profunda, o que vem a refletir diretamente na porosidade total de ambos, onde 0 tratamento com Piatã que obteve maior macroporosidade também teve maior porosidade total, e o tratamento com Ruziziensis por ter apresentado menor macroporosidade obteve menor porosidade total. Marchão et al. (2007) também encontraram resultados que corroboram com os encontrados neste trabalho, onde um menor valor de macroporosidade e porosidade total resultou também em uma maior densidade do solo.

Na Tabela 3, observa-se diferenças claras na distribuição da porcentagem de macroporos nas diferentes épocas de avaliação, na qual a maior porcentagem de macroporos foi obtida na coleta realizada em fevereiro de 2017. Observase na Tabela 4 que os resultados de microporosidade se relacionam com os resultados de macroporosidade, quando a macroporosidade está maior, a microporosidade consequentemente tem um valor menor. Esta característica possivelmente pode estar associada a textura do solo que é arenosa e estudos posteriores serão realizados para investigar este comportamento.

Na coleta de fevereiro de 2017 que foi realizada após a colheita da soja, a distribuição dos poros mudou, diminuindo o número de microporos. Para a interação entre porosidade total e a data de coleta, observa-se que nas duas camadas estudas a porosidade total diminuiu com o passar do tempo de implantação do sistema, com declínio acentuado na última coleta realizada após a colheita do milho (Tabela 5).

Em pesquisa desenvolvida por Souza et al. (2019), foram obtidos resultados semelhantes aos verificados na presente pesquisa, onde a redução nos macroporos refletiu significativamente na porosidade total que também reduziu, efeito esse que acontece geralmente em função do não revolvimento do solo e pisoteio animal. Contrariamente, Nascimento Junior (2017) verificou que o pisoteio animal decorrente do pastejo de entressafra na região do cerrado brasileiro não degradou a estrutura do solo em sistemas de integração agricultura-pecuária, mantendo a estrutura e consequentemente outras propriedades físicas do solo, como a densidade do solo, porosidade total e macroporosidade do solo. 
Tabela 3. Desdobramento da interação significativa entre macroporosidade do solo em função das épocas de coleta. Caiuá - SP. 2016-17.

\begin{tabular}{|c|c|c|c|}
\hline \multicolumn{4}{|c|}{$0,0-0,10 \mathrm{~m}$} \\
\hline \multirow[t]{2}{*}{ Tratamento } & \multicolumn{3}{|c|}{ Data de coleta } \\
\hline & Set/2016 & Fev/2017 & Nov/2017 \\
\hline Soja-Milho+U. ruziziensis & $16,86 \mathrm{AB}$ & $24,04 \mathrm{~A}$ & $10,72 \mathrm{~B}$ \\
\hline Soja-Milho+Piatã & 16,70 & 18,85 & 12,45 \\
\hline Soja-Milho+Paiaguás & $11,08 \mathrm{~B}$ & $26,20 \mathrm{~A}$ & $10,84 \mathrm{~B}$ \\
\hline Soja-Piatã & $15,55 \mathrm{~B}$ & $27,63 \mathrm{~A}$ & $12,95 \mathrm{~B}$ \\
\hline Soja-Paiaguás & $17,05 A B$ & $23,46 \mathrm{~A}$ & $10,82 \mathrm{~B}$ \\
\hline Soja-Milho & $16,35 \mathrm{AB}$ & $20,61 \mathrm{~A}$ & 10,06 B \\
\hline \multicolumn{4}{|c|}{$0,10-0,20 \mathrm{~m}$} \\
\hline \multirow[t]{2}{*}{ Tratamento } & \multicolumn{3}{|c|}{ Data de coleta } \\
\hline & Set/2016 & Fev/2017 & Nov/2017 \\
\hline Soja-Milho+U. ruziziensis & $14,72 \mathrm{~B}$ & 20,96 b A & 9,45 b C \\
\hline Soja-Milho+Piatã & $13,13 \mathrm{~B}$ & 17,44 b A & $1092 a b B$ \\
\hline Soja-Milho+Paiaguás & $10,83 \mathrm{~B}$ & 21,76 b A & $10,73 a b B$ \\
\hline Soja-Piatã & $12,64 \mathrm{~B}$ & 27,12 a $A$ & 15,08 a B \\
\hline Soja-Paiaguás & $14,49 \mathrm{~B}$ & 19,79 b A & 11,99 ab B \\
\hline Soja-Milho & $13,87 \mathrm{~B}$ & 22,54 ab A & $8,30 \mathrm{~b} \mathrm{C}$ \\
\hline
\end{tabular}

Médias seguidas por letras iguais e maiúsculas na linha e minúsculas na coluna não diferem estatisticamente pelo teste Tukey a $5 \%$ de probabilidade.

Tabela 4. Desdobramento da interação significativa entre microporosidade do solo em função das épocas de coleta. Caiuá - SP. 2016-17.

\begin{tabular}{cccc}
\hline & \multicolumn{3}{c}{$\mathbf{0 , 0 - 0 , 1 0 m}$} \\
\hline Tratamento & Set/2016 & Fev/2017 & Nov/2017 \\
\cline { 2 - 4 } & $26,26 \mathrm{~A}$ & $15,28 \mathrm{C}$ & $21,01 \mathrm{~B}$ \\
Soja-Milho+U. ruziziensis & $26,25 \mathrm{~A}$ & $19,52 \mathrm{~B}$ & $21,05 \mathrm{~B}$ \\
Soja-Milho+Piatã & $27,29 \mathrm{~A}$ & $17,06 \mathrm{C}$ & $22,23 \mathrm{~B}$ \\
Soja-Milho+Paiaguás & $26,55 \mathrm{~A}$ & $16,30 \mathrm{~B}$ & $21,10 \mathrm{~B}$ \\
Soja-Piatã & $26,47 \mathrm{~A}$ & $17,95 \mathrm{~B}$ & $22,52 \mathrm{AB}$ \\
Soja-Paiaguás & $27,31 \mathrm{~A}$ & $20,30 \mathrm{~B}$ & $23,20 \mathrm{AB}$ \\
Soja-Milho & $\mathbf{0 , 1 0 - 0 , 2 0 m}$ & & \\
\hline & & Data de coleta & \\
\hline Tratamento & Set/2016 & Fev/2017 & Nov/2017 \\
\cline { 2 - 4 } & $24,18 \mathrm{~A}$ & $17,20 \mathrm{~B}$ & $21,25 \mathrm{AB}$ \\
Soja-Milho+U. ruziziensis & $26,91 \mathrm{~A}$ & $21,22 \mathrm{AB}$ & $20,22 \mathrm{~B}$ \\
Soja-Milho+Piatã & $28,68 \mathrm{~A}$ & $16,95 \mathrm{~B}$ & $22,35 \mathrm{AB}$ \\
Soja-Milho+Paiaguás & $29,16 \mathrm{~A}$ & $14,99 \mathrm{~B}$ & $20,40 \mathrm{~B}$ \\
Soja-Piatã & $28,74 \mathrm{~A}$ & $19,59 \mathrm{~B}$ & $20,24 \mathrm{~B}$ \\
Soja-Paiaguás & $25,24 \mathrm{~A}$ & $18,41 \mathrm{~B}$ & $22,94 \mathrm{AB}$ \\
Soja-Milho & &
\end{tabular}

Médias seguidas por letras iguais e maiúsculas na linha não diferem estatisticamente pelo teste Tukey a $5 \%$ de probabilidade. 
Tabela 5. Desdobramento da interação significativa entre porosidade total do solo em função da épocas de coleta. Caiuá - SP. 2016-17.

\begin{tabular}{|c|c|c|c|}
\hline \multicolumn{4}{|c|}{$0,0-0,10 \mathrm{~m}$} \\
\hline \multirow{2}{*}{ Tratamento } & \multicolumn{3}{|c|}{ Data de coleta } \\
\hline & Set/2016 & Fev/2017 & Nov/2017 \\
\hline Soja-Milho+U. ruziziensis & $43,13 \mathrm{~A}$ & $39,32 \mathrm{~A}$ & $31,74 \mathrm{~B}$ \\
\hline Soja-Milho+Piatã & $42,96 \mathrm{~A}$ & $38,37 \mathrm{AB}$ & $33,50 \mathrm{~B}$ \\
\hline Soja-Milho+Paiaguás & $38,38 \mathrm{AB}$ & $43,27 \mathrm{~A}$ & $33,08 \mathrm{~B}$ \\
\hline Soja-Piatã & $42,11 \mathrm{~A}$ & $43,94 \mathrm{~A}$ & $34,06 \mathrm{~B}$ \\
\hline Soja-Paiaguás & $43,53 \mathrm{~A}$ & $41,42 \mathrm{~A}$ & $33,34 \mathrm{~B}$ \\
\hline Soja-Milho & $43,66 \mathrm{~A}$ & $40,93 \mathrm{~A}$ & $33,27 \mathrm{~B}$ \\
\hline \multicolumn{4}{|c|}{$0,10-0,20 m$} \\
\hline \multirow{2}{*}{ Tratamento } & \multicolumn{3}{|c|}{ Data de coleta } \\
\hline & Set/2016 & Fev/2017 & Nov/2017 \\
\hline Soja-Milho+U. ruziziensis & $38,90 \mathrm{~A}$ & $38,16 \mathrm{~A}$ & $30,70 \mathrm{~B}$ \\
\hline Soja-Milho+Piatã & $40,04 \mathrm{~A}$ & $38,66 \mathrm{~A}$ & $31,13 \mathrm{~B}$ \\
\hline Soja-Milho+Paiaguás & $39,52 \mathrm{~A}$ & $38,71 \mathrm{AB}$ & $33,08 \mathrm{~B}$ \\
\hline Soja-Piatã & $41,80 \mathrm{~A}$ & $42,12 \mathrm{~A}$ & $35,48 \mathrm{~B}$ \\
\hline Soja-Paiaguás & $43,23 \mathrm{~A}$ & $39,38 \mathrm{~A}$ & $32,23 \mathrm{~B}$ \\
\hline Soja-Milho & $39,11 \mathrm{~A}$ & $40,95 \mathrm{~A}$ & $31,25 \mathrm{~B}$ \\
\hline
\end{tabular}

Médias seguidas por letras iguais e maiúsculas na linha não diferem estatisticamente pelo teste Tukey a $5 \%$ de probabilidade.

Tabela 6. Desdobramento da interação significativa entre densidade do solo em função da épocas de coleta. Caiuá - SP. 2016-17.

\begin{tabular}{|c|c|c|c|}
\hline \multicolumn{4}{|c|}{$0,0-0,10 \mathrm{~m}$} \\
\hline \multirow{2}{*}{ Tratamento } & \multicolumn{3}{|c|}{ Data de coleta } \\
\hline & Set/2016 & Fev/2017 & Nov/2017 \\
\hline Soja-Milho+U. ruziziensis & 1,54 & 1,65 & 1,65 \\
\hline Soja-Milho+Piatã & $1,54 \mathrm{~B}$ & $1,67 \mathrm{~A}$ & $1,62 \mathrm{AB}$ \\
\hline Soja-Milho+Paiaguás & 1,61 & 1,57 & 1,66 \\
\hline Soja-Piatã & 1,54 & 1,57 & 1,59 \\
\hline Soja-Paiaguás & 1,49 & 1,60 & 1,60 \\
\hline Soja-Milho & 1,51 & 1,59 & 1,57 \\
\hline \multicolumn{4}{|c|}{$0,10-0,20 m$} \\
\hline \multirow{2}{*}{ Tratamento } & \multicolumn{3}{|c|}{ Data de coleta } \\
\hline & Set/2016 & Fev/2017 & Nov/2017 \\
\hline Soja-Milho+U. ruziziensis & 1,64 & 1,66 & 1,67 \\
\hline Soja-Milho+Piatã & 1,59 & 1,66 & 1,66 \\
\hline Soja-Milho+Paiaguás & 1,59 & 1,63 & 1,67 \\
\hline Soja-Piatã & 1,59 & 1,59 & 1,56 \\
\hline Soja-Paiaguás & 1,56 & 1,65 & 1,65 \\
\hline Soja-Milho & 1,61 & 1,63 & 1,66 \\
\hline
\end{tabular}

Médias seguidas por letras iguais e maiúsculas na linha não diferem estatisticamente pelo teste Tukey a $5 \%$ de probabilidade. 
Figura 1. Componentes do solo Set/2016

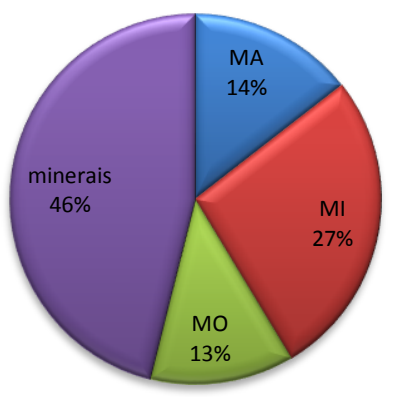

Figura 2. Componentes do solo Fev/2017

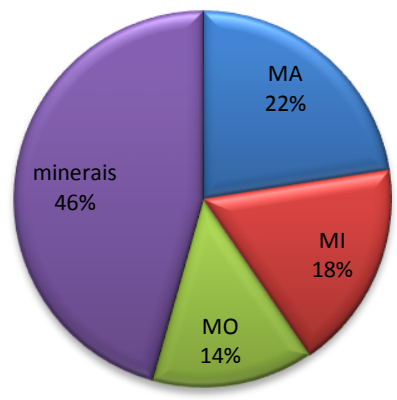

Figura 3. Componentes do solo Nov/2017

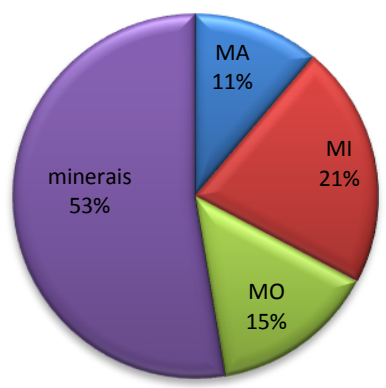

Observa-se um elevado valor de densidade do solo no consórcio Soja-Milho+Piatã $\left(1,67 \mathrm{~g} \mathrm{~cm}^{-3}\right)$ na camada 0,0-0,10m (Tabela 6). Bonini et al. (2016), verificaram valores semelhantes de densidade em solo sobre sistema de plantio direto, em que as maiores densidades foram encontradas onde se tinha $o$ menor volume de macroporos. Quando se compara os valores de densidade da caracterização física da área realizada em 2015 (Tabela 1) com os valores de densidade atuais pode-se observar um pequeno aumento no valor deste atributo. Contudo, segundo trabalho realizado por Stefanoski et al. (2013), constataram que com o passar de muitos anos de implantação do sistema, a densidade do solo sob plantio direto tende a diminuir, devido, em grande parte, ao aumento do teor de matéria orgânica na camada superficial, melhorando a estrutura do solo por completo.

No presente trabalho foi possível observar as mudanças evidentes na distribuição dos poros ao longo de uma safra agrícola, o que acontece muito em função das diferenças entre os sistemas radiculares das plantas e do manejo empregado dentro do sistema produtivo. (DENARDIN et al., 2012; STEFANOSKI et al., 2013; ROSSI NETO et al., 2018). As figuras 1,2 e 3 mostram também como variou cada componente do solo estudado. Apesar da porosidade total da área ter diminuído quando comparado os valores da primeira e última coleta, deve-se levar em conta que os valores de matéria orgânica aumentaram, e como já dito anteriormente, isso pode influenciar de forma positiva na estrutura do solo em geral, reduzindo assim a densidade do solo com o passar dos anos. Em sua maioria, os estudos sobre o efeito de sistemas de manejo têm demonstrado que as alterações no conteúdo de matéria orgânica são lentas, necessitando de um período de tempo relativamente longo para serem detectadas (SOUZA et al., 2019).

\section{Conclusões}

Os sistemas integrados de produção agropecuária modificaram positivamente a distribuição de macro e microporos em função da rotação de cultura e momento da obtenção dos dados (2017) em relação a caracterização inicial (2016).

Os tratamentos soja+piatã e soja+milho+piatã foram os tratamentos com maior macroporosidade e menor densidade do solo.

\section{Agradecimentos}

Agradecemos a Fundação de Amparo a Pesquisa do Estado de São Paulo pela concessão da bolsa de iniciação cientifica (Proc FAPESP 2017/17300-8), ao CNPQ pelo auxilio a pesquisa (PROC CNPq UNIVERSAL 431914/2018-4 Chamada MCTIC/CNPq № 28/2018), ao Grupo Facholi por ceder a área e infraestrutura para realização do experimento, e também a Faculdade de Ciências Agrárias e Tecnológicas pela estrutura de laboratórios para condução das análises.

\section{Referências}

ALVARES C.A., STAPE J.L., SENTELHAS P.C., DE MORAES GONCALVES J.L., SPAROVEK G. Köppen's climate classification map for Brazil. Meteorologische, v. 22, n. 6, p. 711-728. 2013. http://dx.doi.org/10.1127/0941-2948/2013/0507 
ASSMANN, T. S.; RONZELLI JÚNIOR, P.; MORAES, A.; ASSMANN, A. L.; KOEHLER, H. S.; SANDINI, I. Rendimento de milho em área de integração lavoura-pecuária sob o sistema plantio direto, em presença e ausência de trevo branco, pastejo e nitrogênio. Revista Brasileira de Ciência do Solo, v.27, n.4, p.675-683, 2003. http://dx.doi.org/10.1590/S0100$\underline{06832003000400012}$

BONINI, C. S. B., LUPATINI, G. C., ANDRIGHETTO, C., MATEUS, G. P., HEINRICHS, R., ARANHA, A. S., SANTANA, E. A. R. \& MEIRELLES, G. C. Produção de forragem e atributos químicos e físicos do solo em sistemas integrados de produção agropecuária. Pesquisa Agropecuaria Brasileira, v.51, n.9, p.1695-1698. 2016. http://dx.doi.org/10.1590/s0100-

\section{$\underline{204 \times 2016000900070}$}

CARVALHO, P. C. F.; ANGHINONI, I.; MORAES, A.; SOUZA, E. D.; SULC, R. M.; LANG, C. R.; FLORES, J. P. C.; LOPES, M. L. T.; SILVA, J. L. S.; CONTE, O.; WESP, C. L.; LEVIEN, R.; FONTANELI, R. S.; BAYER, $C$. Managing grazing animals to achieve nutrient cycling and soil improvement in no-till integrated systems. Nutrient Cycling in Agroecosystems, v.87, n.2, p.259-273, 2010. http://dx.doi.org/10.1007/s10705-010-9360-x

CERVELATI, K.F.; SOUSA NETO, E. L.; EGUCHI, E. S.; SILVA, M. R.; PIERANGELI, M. A. P. Efeito de diferentes sistemas de pastejo em atributos físicos do solo. PUBVET - Publicações em Medicina Veterinária e Zootecnia, v.5, n.22, p.1142, 2011.

https://doi.org/10.22256/pubvet.v5n22.1142

CHIODEROLI C.A., MELLO L.M.M., GRIGOLLI P.J., FURLANI C.E.A., SILVA J.O.R., CESARIN A.L. Atributos físicos do solo, produtividade de soja em sistema de consórcio milho, braquiária. Revista Brasileira de Engenharia Agrícola e Ambiental, v.16, n.1, p.37-43, 2012. http://dx.doi.org/10.1590/S1415-

$\underline{43662012000100005}$

CHIODEROLI, C. A; MELLO, L. M. M.; GRIGOLLI, P. J.; SILVA, J. O. R.; CESARIN, A. L. Consorciação de braquiárias com milho outonal em plantio direto sob pivô central. Engenharia Agrícola, v.30, n.6, p.1101-1109, 2010. http://dx.doi.org/10.1590/S0100$\underline{69162010000600011}$
COSTA, N. R.; ANDREOTTI, M.; CRUSCIOL, C. A. C.; PARIZ, C. M.; LOPES, K. S. M.; YOKOBATAKE, K. L. A.; FERREIRA, J. P.; LIMA, C. G. R.; SOUZA, D. M. Effect of intercropped tropical perennial grasses on the production of sorghum-based silage. Agronomy Journal, v.108, n.1, p.2379-2390, 2016.

https://doi.org/10.2134/agronj2016.07.0385

COSTA, N. R.; ANDREOTTI, M.; LOPES, K. S. M.; YOKOBATAKE, K. L.; FERREIRA, J. P.; PARIZ, C. M.; BONINI, C. S. B.; LONGHINI, V. Z. Atributos do solo e acúmulo de carbono na integração lavourapecuária em sistema plantio direto. Revista Brasileira de Ciência do Solo, v.39, n.3, p.852863, 2015. http://dx.doi.org/10.1590/01000683rbcs2014026 $\underline{9}$

DENARDIN, J. E.; KOCHHANN, R. A.; FAGANELLO, A.; SANTI, A.; DENARDIN, N. A.; WIETHÖLTER, S. Diretrizes do sistema plantio direto no contexto da agricultura conservacionista. Brasília: Embrapa Trigo, 2012.

FERREIRA, D.F. Sisvar: a computer statistical analysis system. Ciência e Agrotecnologia, v.35, n.6, p.1039-1042, 2011. http://dx.doi.org/10.1590/S1413$\underline{70542011000600001}$

FLORES, J. P. C.; ANGHINONI, I.; CASSOL, L. C.; CARVALHO, P. C. F.; LEITE, J. G. D. B.; FRAGA, T. I. Atributos físicos do solo, rendimento de soja em sistema plantio direto em integração lavourapecuária com diferentes pressões de pastejo. Revista Brasileira de Ciência do Solo, v.31, n.4, p.771-80, 2007. http://dx.doi.org/10.1590/S0100$\underline{06832007000400017}$

LANZANOVA, M.E.; NICOLOSO, R. S.; LOVATO, T.; ELTZ, F.L.F.; AMADO, T.J.C.; REINERT, D.J. Atributos físicos do solo em sistema de integração lavoura-pecuária sob plantio direto. Revista Brasileira de Ciência do Solo, v.31, n.5, p.1131-1140, 2007. http://dx.doi.org/10.1590/S0100$\underline{06832007000500028}$

MACEDO, M. C. M. Integração lavoura e pecuária: - estado da arte e inovações tecnológicas. Revista Brasileira de Zootecnia, v.38, (supl. esp.), 
p.133-146, 2009. https://doi.org/10.1590/S1516$\underline{35982009001300015}$

MARCHÃO, R. L.; BALBINO, L. C.; SILVA, E. M.; SANTOS JUNIOR, J. D. G.; SÁ, M. A. C.; VILELA, L.; BECQUER T. Qualidade física de um Latossolo Vermelho sob sistemas de integração lavourapecuária no Cerrado. Pesquisa Agropecuária Brasileira, v.42, n.6, p.873-882, 2007. http://dx.doi.org/10.1590/S0100$\underline{204 \times 2007000600015}$

NASCIMENTO JÚNIOR, L. F. Modelagem e quantificação da compactação promovida pelo pisoteio animal em um latossolo submetido ao sistema de integração agricultura-pecuária. 2017. Dissertação (Mestrado em Agronomia) Instituto Federal de Educação, Ciência e Tecnologia Goiano, Campus Rio Verde, 2017.

PARIZ, C. M.; COSTA, C.; CRUSCIOL, C. A. C.; MEIRELLES, P. R. L.; CASTILHOS, A. M.; ANDREOTTI, M.; COSTA, N. R.; MARTELLO, J. M.; SOUZA, D. M.; PROTES, V. M.; LONGHINI, V. Z.; FRANZLUEBBERS, A. J. Production, nutrient cycling and soil compaction to grazing of grass companion cropping with corn and soybean. Nutrient Cycling in Agroecosystems, v.107, n.1, p.1-20, 2017. https://doi.org/10.1007/s10705$\underline{016-9821}$

PRADO, H. Pedologia fácil: aplicações em solos tropicais. Piracicaba: Helio do Prado, 2013.

ROSSI NETO, J.; DE SOUZA, Z. M.; KÖLLN, O. T.; CARVALHO, J. L. N.; FERREIRA, D. A.; CASTIONI, G. A. F.; BARBOSA, L. C.; DE CASTRO, S. G. Q.; BRAUNBECK, O. A.; GARSIDE, A. L.; FRANCO, H. C. $J$. The Arrangement and Spacing of Sugarcane Planting Influence Root Distribution and Crop Yield. BioEnergy Research, v.11, p.291-304, 2018. https://doi.org/10.1007/s12155-018-9896-1

SANTOS, R. S. M.; OLIVEIRA, I. P.; MORAIS, R. F.; URQUIAGA, S. C.; BODDEY, R. M.; ALVES, B. J. R. Componentes da parte aérea, raízes de pastagens de Brachiaria spp em diferentes idades após a reforma, como indicadores de produtividade em ambiente de Cerrado. Pesquisa Agropecuária Tropical, v.37, n.2, p.119-124, 2007.

SANTOS, H. G. dos; JACOMINE, P. K. T.; ANJOS, L. H. C.; OLIVEIRA, V. A.; LUMBRERAS, J. F.; COELHO, M. R.; ALMEIDA, J. A.; ARAUJO FILHO, J. C.;
OLIVEIRA, J. B.; CUNHA, T. J. F. Sistema de Classificação de solos. Brasília: Embrapa Solos, 2018.

SARMENTO, P.; RODRIGUES, L. R. A.; LUGÃO, S. M. B.; CRUZ, M. C. P.; CAMPOS, F. P.; FERREIRA, M. E.; OLIVEIRA, R. F. Sistema radicular do Panicum maximum Jacq. CV. IPR-86 Milênio adubado com nitrogênio, submetido à lotação rotacionada. Revista Brasileira de Zootecnia, v.37, n.1, p.27-34, 2008. http://dx.doi.org/10.1590/S1516$\underline{35982008000100004}$

SOUZA, J.F.D.; PERUSSO, R. L. S.; BONINI, C. S. B.; SOUZA, C. T.; LUPATINI, G. C.; ANDRIGHETTO, C.; MATEUS, G. P.; PEDRO, F. G. ATRIBUTOS FÍSICOS, MATÉRIA ORGÂNICA DO SOLO E PRODUÇÃO DE CAPIM MARANDU EM SISTEMA DE INTEGRAÇÃO LAVOURA-PECUÁRIA-FLORESTA. Brazilian Journal of Biosystems Engineering, v. 13, n.1, p.51-64, 2019.

https://doi.org/10.18011/bioeng2019v13n1p51$\underline{64}$

SPERA, S. T.; SANTOS, H. P.; FONTANELI, R. S. \& TOMM, G. O. Integração lavoura e pecuária e os atributos físicos de solo manejado sob sistema plantio direto. Revista Brasileira de Ciência do Solo, v.33, n.1, p.129-136, 2009. http://dx.doi.org/10.1590/S0100$\underline{06832009000100014}$

SPERA. S. T.; SANTOS. H. P.; FONTANELI. R. S.; TOMM. G. O. Efeitos de sistemas de produção de grãos envolvendo pastagens sob plantio direto nos atributos físicos de solo e na produtividade. Revista Brasileira de Ciência do Solo, v.28, n.3, p.533-542.

2004.

http://dx.doi.org/10.1590/S0100-

$\underline{06832004000300014}$

STEFANOSKI, D. C.; SANTOS, G. G.; MARCHÃO, R. L.; PETTER, F. A. \& PACHECO, L. P. Uso e manejo do solo e seus impactos sobre a qualidade física. Revista Brasileira de Engenharia Agrícola e Ambiental, v.17, n.12, p.1301-1309, 2013. http://dx.doi.org/10.1590/S141543662013001200008.

TEIXEIRA, P. C.; DONAGEMMA, G. K.; FONTANA, A. Manual de métodos de análise de solo. Brasília: Embrapa Solos, 2017. 